\title{
Statistical Behavior of Formation Process of Magnetic Vortex State in $\mathrm{Ni}_{80} \mathrm{Fe}_{20}$ Nanodisks
}

\author{
Mi-Young Im ${ }^{\text {* }}$, Peter Fischer1, ${ }^{1}$ amada Keisuke ${ }^{2}$, and Shinya Kasai ${ }^{2,3}$ \\ ${ }^{1}$ Center for X-ray Optics, Lawrence Berkeley National Laboratory, Berkeley \\ CA94720, USA \\ ${ }^{2}$ Institute for Chemical Research, Kyoto University, Uji 611-0011, Japan \\ ${ }^{3}$ Spintronics Group, Magnetic Material Center, National Institute for Materials Science \\ (NIMS), Tukuba 305-0047, Japan
}

Magnetic vortices in magnetic nanodots, which are characterized by an in-plane (chirality) and an out-of-plane (polarity) magnetizations, have been intensively attracted because of their high potential for technological application to data storage and memory scheme as well as their scientific interest for an understanding of fundamental physics in magnetic nanostructures [1]. Complete understanding of the formation process of vortex state in magnetic vortex systems is very significant issue to achieve storage and memory technologies using magnetic vortices and understand intrinsic physical properties in magnetic nanostructures. In our work, we have statistically investigated the formation process of vortex state in permalloy $\left(\mathrm{Py}, \mathrm{Ni}_{80} \mathrm{Fe}_{20}\right)$ nanodisks through the direct observation of vortex structure utilizing a magnetic transmission soft X-ray microscopy (MTXM) with a high spatial resolution down to $20 \mathrm{~nm}$ [2]. Magnetic imaging in Py nanodots was performed at the $\mathrm{Fe}_{3}(707 \mathrm{eV})$ absorption edge. Figure 1 shows in-plane and out-of-plane magnetic components observed in $40 \mathrm{~nm}$ thick nanodot arrays with different dot radius of $\mathrm{r}=500$ and $400 \mathrm{~nm}$, respectively. Vortex chirality, either clockwise (CW) or counter-clockwise (CCW), and polarity, either up or down, are clearly visible in both arrays. To investigate the statistical behavior in formation process of the vortex state, the observation of vortex structure at a remanant state after saturation of nanodots by an external magnetic field of $1 \mathrm{kOe}$ has been repeatedly performed over 100 times for each array. The typical MTXM images of vortex chirality taken in two successive measurements together with their overlapped images in nanodot arrays of $r=500$ and 400 $\mathrm{nm}$ are displayed in Fig. 2. Within the statistical measurement, the formation process of chirality of either $\mathrm{CW}$ or $\mathrm{CCW}$ is quite stochastic in each nanodot. Similar behavior is also witnessed in the formation of vortex polarity observed in consecutive experiments of the same arrays. Interestingly, a particular selectivity between the circulation sense of chirality and orientation sense of polarity for each other is found in the formation process of vortex state despite of their respective stochastic generation in repeated measurements. Dzyaloshinskii-Moriya (D-M) interaction in magnetic nanodisks, which is inevitably generated due to the breaking of inversion symmetry at surface/interface in magnetic thin layers [3], is mainly responsible for the experimentally witnessed selectivity between chirality and polarity in a formation of vortex structure.

This work was supported by the Director, Office of Science, Office of Basic Energy Sciences, Materials Sciences and Engineering Division, of the U.S. Department of Energy under Contract No. DE-AC02-05CH11231. 
[1] T. Shinjo, T. Okuno, R. Hassdorf, K. Shigeto, and T. Ono, Science 289, 930(2000).

[2] P. Fischer, AAPPS bulletin 18, 12 (2008).

[3] A.B. Butenko, A.A. Leonov, A. N. Bogdanov, and U. K. Rößler, Phys. Rev. B 80, 134410 (2009).

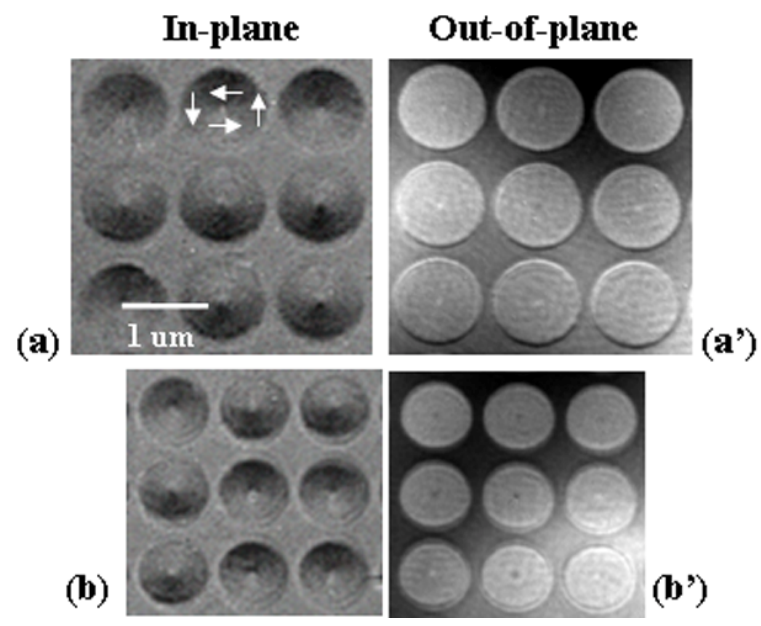

Fig. 1. MTXM images of in-plane and out-of-plane magnetizations observed in $40 \mathrm{~nm}$ thick Py nanodot arrays with different radius of $r=500$ and $400 \mathrm{~nm}$. Here, the relatively dark (bright) contrast in Figs. 1(a) and (b) indicates the magnetization directed to left (right) and spot in the center of dots in Figs. 1(a') and (b') corresponds to the upward (downward) core magnetization.

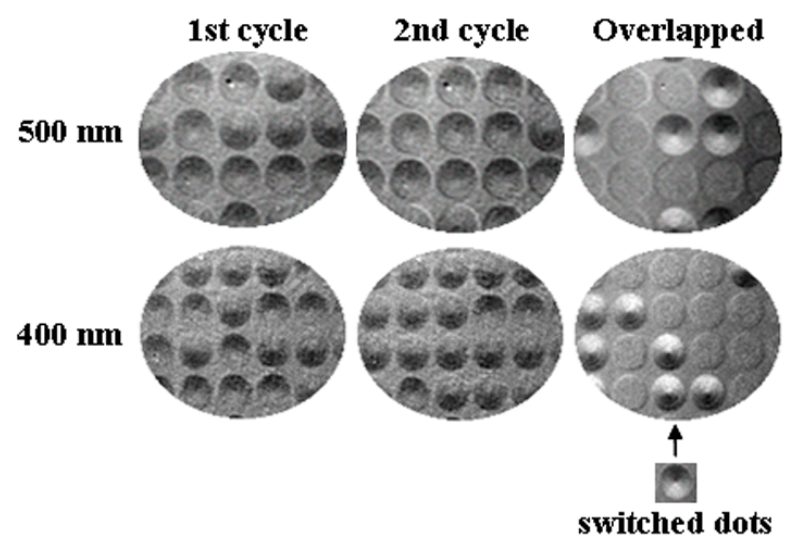

Fig. 2. Images of vortex chirality taken in two successive measurements together with their overlapped images in Py nanodot arrays of $\mathrm{r}=500$ and $400 \mathrm{~nm}$. In overlapped images, clear vortex structure revealed in some nanodots indicates that the circulation sense of the vortex chirality in those nanodots is switched in repetitions. 


\section{DISCLAIMER}

This document was prepared as an account of work sponsored by the United States Government. While this document is believed to contain correct information, neither the United States Government nor any agency thereof, nor the Regents of the University of California, nor any of their employees, makes any warranty, express or implied, or assumes any legal responsibility for the accuracy, completeness, or usefulness of any information, apparatus, product, or process disclosed, or represents that its use would not infringe privately owned rights. Reference herein to any specific commercial product, process, or service by its trade name, trademark, manufacturer, or otherwise, does not necessarily constitute or imply its endorsement, recommendation, or favoring by the United States Government or any agency thereof, or the Regents of the University of California. The views and opinions of authors expressed herein do not necessarily state or reflect those of the United States Government or any agency thereof or the Regents of the University of California. 